\title{
An Artificial Neural Network Approach for Predicting Kinematics in Handball Throws
}

\author{
Abdel-Rahman Ibrahim Akl ${ }^{1, ~ *, ~ A m r ~ A b d u l f a t t a h ~ H a s s a n ~}{ }^{2,3}$ \\ ${ }^{1}$ Faculty of Physical Education (Abo Qir), Alexandria University, Alexandria, Egypt \\ ${ }^{2}$ Department of Sports Training, Faculty of Sports Education, Mansoura University, Mansoura, Egypt \\ ${ }^{3}$ Institute of Sport Science, University Graz, Austria
}

Email address:

abdelrahman.akl@alexu.edu.eg (Abdel-Rahman I. A.), amrahh@mans.edu.eg (Amr A. H.)

${ }^{*}$ Corresponding author

\section{To cite this article:}

Abdel-Rahman Ibrahim Akl, Amr Abdulfattah Hassan. An Artificial Neural Network Approach for Predicting Kinematics in Handball Throws. American Journal of Sports Science. Vol. 5, No. 5, 2017, pp. 35-39. doi: 10.11648/j.ajss.20170505.13

Received: February 26, 2017; Accepted: April 19, 2017; Published: October 26, 2017

\begin{abstract}
The purpose of this study was to test a new method to predict the kinematics of center of mass (COM) during the take-off phase of the handball shot by mean of multilayer perceptron neural networks (MLPs) based on data from only the force platform. Ten trials' of handball jump shot data from the force platform were obtained. The kinetic data of jump shot trials (force, impulse, and work) were used to feed the net and the data from the force platform kinematics (acceleration, velocity, and displacement) was used to evaluate the production data of the MLP neural network model. A commercial artificial neural network software was used to predict the target kinematic parameters (NeuroDimension, 2014®). The Pearson correlations of all Kinetics parameters between the original and production data was $>0.99$. The MLPs model successfully predicted the target kinematics depending on kinetics in the handball jump shot under the conditions of this study.
\end{abstract}

Keywords: Neural Networks, Biomechanics, Prediction

\section{Introduction}

In sports, players choose their actions based on the game situation. Handball players usually have constantly evolving skills and tactics in various game situations. Therefore, they need to obtain high levels of coordination of their movements to run, jump, push, change direction, pass, catch, shoot, check and block [1]. Thus, determining the factors that influence performance in team handball based on scientific studies would be a valuable contribution towards increasing the quality of training for coaches and practitioners; in addition to develop specific measuring methods and tests for scientific studies [1].

In handball competition, $73-75 \%$ of all throws are jump throws, [2]. Gorostiaga et al. (2005) analyzed the performances of team handball players in different levels and concluded that age and body weight contributed significantly to differences in ball release speed during throws [3]. And Wagner et al. (2010) found significant differences in the ball release speed in jump throws, in addition to differences in body height and weight across various performance levels [4]. These studies suggest that jump height for throws may be considered one of the important parameters in team handball. A high jump offers a good position to throw over the block of the rival defender. This happens when players shoot from the back court position or have more time for a shot (an increase in flight time) and when attempting to react the goalkeeper actions [1].

On the other hand, the center of mass (COM) excursions yield the best conditions for mastering balance and keeping the lower body more stable and effective. Additionally, the maximum change in COM velocity is considered an important parameter that is potentially related to performance [5]. The force of COM considers relevant information that exists while performing sport techniques [6].

Models may be used to address the forward and inverse dynamic problems in human movement. In forward dynamic problems, the driving forces are specified and the problem is to determine the kinematics of motion. In the inverse dynamic problems, the kinematics of motion are specified and the 
problem is to determine the driving forces that produced the motion [7, 8].

Several computer simulation methods have been proposed in order to model human motion [9]. First, kinematic models consist of defining a mathematical expression to represent trajectories as a function of time [10]. Second, dynamics are used to ensure that the resulting motions verify the mechanical laws [11]. Third, motion capture and motion modification have been widely used by computing a new motion in the neighbourhood of the original one [12].

The biomechanical model is a structure that represents the relationship between the objectives of the skills and the factors that produce those skills [13]. Laboratory systems were designed to measure more dimensions with greater precision, but these methods are complex and expensive [14]. Therefore, artificial neural networks (ANNs) can be likened to a flexible mathematical function that may have many configurable internal parameters. Once the neural network is trained, it can give new productions that have not previously been handled and they can attempt to predict new values as accurate output [15].

Previous studies used ANNs in the constructive analysis of biomechanics, which is considered more practical due to recent advances in technology [16]. But the use of ANNs in biomechanical analysis techniques still remains in its infancy [17]. Recently, studies have shown that ANNs may be fit tools for prediction in sports as well as biomechanics, for example the use of ANN to predict the hip, knee, and ankle sagittal moments during a vertical jump by using the output data from a force platform [18-22].

The output data from the neural network offer a nonlinear method for mapping the difficult-to define relationships between kinematics and kinetics parameters. They differ in input data used, the number of hidden layers, training data and other adjustable parameters.

The standard feed forward neural networks for the representation of the mutual effect of this relationship have been used in some studies [23, 24]. ANNs have been widely and successfully used for generalizing the relationships between complex inputs and outputs in a large number of applications in studies of human locomotion, motor control and motor behavior [15, 25-30]. Therefore, it might be possible to design an ANN to predict the kinematic parameters of COM during handball throws using only a force platform output data. In this study, we explored the use of the artificial neural networks to provide a new method for predicting the kinematic parameters of COM during handball throws.

One of the ANNs is the Multilayer Perceptron (MLP) that uses feed forward layers based on static back propagation training. This network is widely used in applications requiring static pattern classification. However, the previous studies did not figure out the use of ANNs to predict data through kinematics and kinetics analysis.

Thus, the purpose of this study was to test a new method for predicting the kinematic parameters of COM during the handball throws by means of MLP neural network based on the output data from a force platform.

\section{Methods}

\subsection{Subjects}

Ten male high level handball players participated in this study (age: $20.9 \pm 1.2$ years; body mass: $82.4 \pm 7.53 \mathrm{~kg}$; height: $188.2 \pm 6.22 \mathrm{~cm}$; experience: $8.2 \pm 1.03$ years). They were part of a professional team that plays in the Egyptian Handball Super League. The players consent was obtained. This study was approved by the institutional ethics committee of studies and research.

\subsection{Protocol}

A 15-minute warm-up which included general and shoulder-specific mobility exercises, as well as stretch exercises and familiarization throws, were required before players' attemps. The participant completed jump-throws from the 7-m penalty line using a standard men's handball after three running steps. A total of five successful attemps were recorded for each participant, with one minute rest between attemps; with the best attemp for each participant was selected for analysis [31, 32].

\subsection{Kinetic and Kinematic Data}

The kinetic and kinematic data during each jump shot trial were calculated. The subject performed the jump shot trials on a strain-gage force platform (MP4060®, Bertec Corporation, Columbus, OH, USA), which measured the ground reaction force (GRF). The data were sampled using an analog-to digital converter at a rate of $1000 \mathrm{~Hz}$. An x, y, z orthogonal coordinate system was used to describe the forces $(F)$ relative to the force plate. The sign convention designated vertical upward $(\mathrm{Fz})$, lateral $(\mathrm{Fx})$, and anterior $(\mathrm{Fy})$ motions. In the next step, Newton's second law was used to calculate the acceleration via the following equation: $\mathrm{a}=\mathrm{F} / \mathrm{m}$, when, $\mathrm{a}=$ acceleration, $\mathrm{F}=$ Force, and $\mathrm{m}=$ mass.

The velocity and displacement were calculated using the integration of acceleration; the integration was calculated via the data analysis and graphing software (OriginPro 8.5 SR1). These data were the lateral force (Force $x$ ), anterior force (Force y), vertical force upward (Force $\mathrm{z}$ ), and magnitude (Force mag), lateral impulse (Impulse $\mathrm{x}$ ), anterior impulse (Impulse y), vertical impulse upward (Impulse $\mathrm{z}$ ), and magnitude (Impulse mag), lateral work (Work x), anterior work (Work y), vertical work upward (Work z), and magnitude (Work mag), as well as the target kinematic parameters in each dataset test (lateral displacement (Displacement $\mathrm{x}$ ), anterior displacement (Displacement y), vertical displacement upward (Displacement $\mathrm{z}$ ), and magnitude (Displacement mag), lateral velocity (Velocity x), anterior velocity (Velocity y), vertical velocity upward (Velocity z), and magnitude (Velocity mag), lateral acceleration (Acceleration $\mathrm{x}$ ), anterior acceleration (Acceleration y), vertical acceleration upward (Acceleration z), and magnitude (Acceleration 
mag).

\subsection{Artificial Neural Network Data}

Artificial neural network software was used to predict the target kinematic parameters of COM during the take-off phase of the handball shot (NeuroDimension, 2014®). Therefore, a

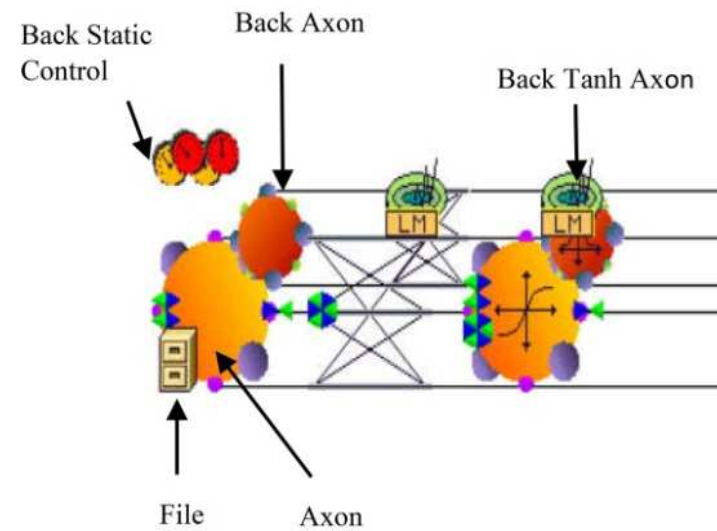

Figure 1. MLPs neural network breadboard.

The net was fed with 159 datasets representing the kinematic data which included $70 \%$ of all data sets collected. In all, $15 \%$ of collected data was used as cross validation. Cross validation processes the lapse in a test set while the system is being prepared with the preparation set. It was realized that the mean squared normalized error (MSNE) would continue to diminish in the preparation set; however, it may begin to increase in the test set [23]. The other data that represented target kinematic parameters (displacement, velocity and acceleration) were marked as desired, and this was also $15 \%$ of the total data.

The maximum epoch was 1000; this field specifies how many iterations (over the training set) would be done if no other criterion took effect. The mean squared error (MSE) is the parameter that terminates the training. The networks were trained one time in each predictive trial. The value of the MSE is the parameter for determining the fitness of the algorithm for prediction, so the result of the training was deemed acceptable when this value was lower than other MSE values during training iterations; it can show through early stopping of the network training (Figure 2).

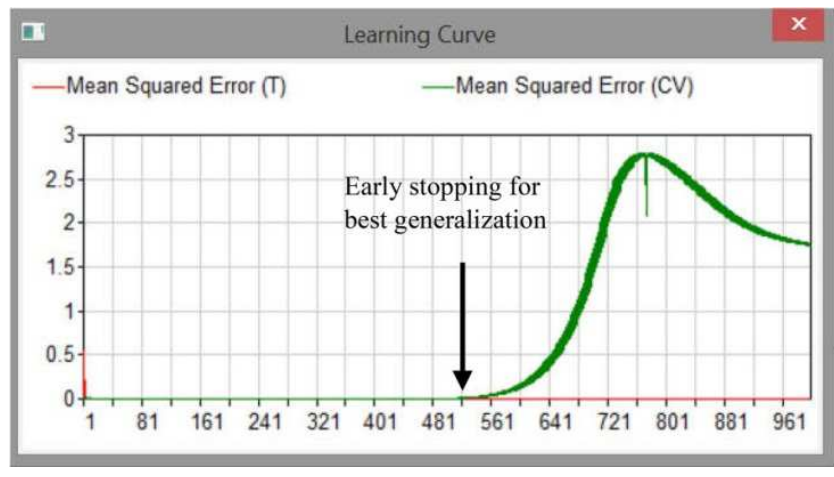

Figure 2. Cross validation (early stopping) during learning curve. multilayer perceptron breadboard (MLPs) was established . The MLPs contained 12 processing elements (PEs), one output PE represents the target kinetic parameter, one hidden layer, and the option of output optimization was the "greedy search" (Figure 1).

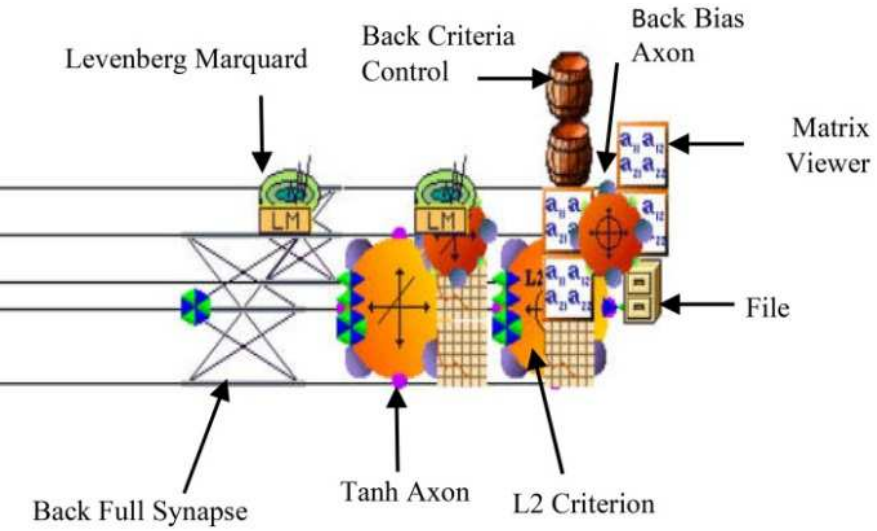
weights (those that had the lowest level of error during the training) were loaded. Then, the MLPs were ready to run the test data. The option to "test network" was then selected. The test window contained different options, and thus, the regression options must be marked before running the test. The output data may be displayed in a text file, on the screen, or in an Excel spreadsheet containing the test report and test production.

\subsection{Statistical Analyses}

Pearson correlation was used to determine the relation between MLP neural network output data and the original analysis data. The mean values of different type of MLP neural network errors (Root mean square error (RMSE), Normalized root mean square error (NRMSE), Mean Absolute Error (MAE), Minimum Absolute Error, and Maximum Absolute Error) were used to determine the fit of prediction algorithm model. It could summarize performance in ways that disregarded the direction of over- or under-prediction, measuring how close predictions were to the eventual outcomes. Standard deviation as root-mean-square error also was calculated, as it represented the sample standard deviation of the differences between the neural network output values (kinematic parameters) and the original values.

\section{Results}

Table 1 shows the average of Pearson correlation (R) between the neural network output values (kinematic parameters) and the input values. It was 0.99 in all parameters. The mean values of different error types were also determined to measure differences between the neural network output values of the (kinematic parameters) and the original values. 
Table 1. Correlation coefficient between MLPs output data and the original analysis data and of several error types also shown.

\begin{tabular}{llllllll}
\hline Kinematic parameter & Units & RMSE & NRMSE & MAE & MINAE & MAXAE & R \\
\hline Displacement $x$ & $\mathrm{~m}$ & 0.003 & 0.046 & 0.002 & 0.001 & 0.006 & 0.998 \\
Displacement y & $\mathrm{m}$ & 0.010 & 0.113 & 0.008 & 0.001 & 0.022 & 0.995 \\
Displacement z & $\mathrm{m}$ & 0.003 & 0.044 & 0.002 & 0.001 & 0.006 & 0.997 \\
Displacement magnitude & $\mathrm{m}$ & 0.079 & 0.157 & 0.069 & 0.000 & 0.114 & 0.990 \\
Velocity x & $\mathrm{m} / \mathrm{s}$ & 0.010 & 0.009 & 0.008 & 0.001 & 0.022 & 0.998 \\
Velocity y & $\mathrm{m} / \mathrm{s}$ & 0.007 & 0.002 & 0.006 & 0.001 & 0.015 & 0.999 \\
Velocity z & $\mathrm{m} / \mathrm{s}$ & 0.050 & 0.021 & 0.046 & 0.017 & 0.083 & 0.999 \\
Velocity magnitude & $\mathrm{m} / \mathrm{s}$ & 0.038 & 0.016 & 0.034 & 0.009 & 0.068 & 0.999 \\
Acceleration x & $\mathrm{m} / \mathrm{s}^{2}$ & 0.581 & 0.020 & 0.420 & 0.012 & 1.370 & 0.997 \\
Acceleration z & $\mathrm{m} / \mathrm{s}^{2}$ & 0.560 & 0.013 & 0.528 & 0.120 & 0.750 & 0.993 \\
Acceleration y & $\mathrm{m} / \mathrm{s}^{2}$ & 1.465 & 0.052 & 1.178 & 0.080 & 2.947 & 0.994 \\
Acceleration magnitude & $\mathrm{m} / \mathrm{s}^{2}$ & 0.934 & 0.030 & 0.840 & 0.198 & 1.522 & 0.999 \\
\hline
\end{tabular}

Notes. RMSE: Root mean square error, NRMSE: Normalized root mean square error, MAE: Mean Absolute Error, MINAE: Minimum Absolute Error, MAXAE: Maximum Absolute Error.
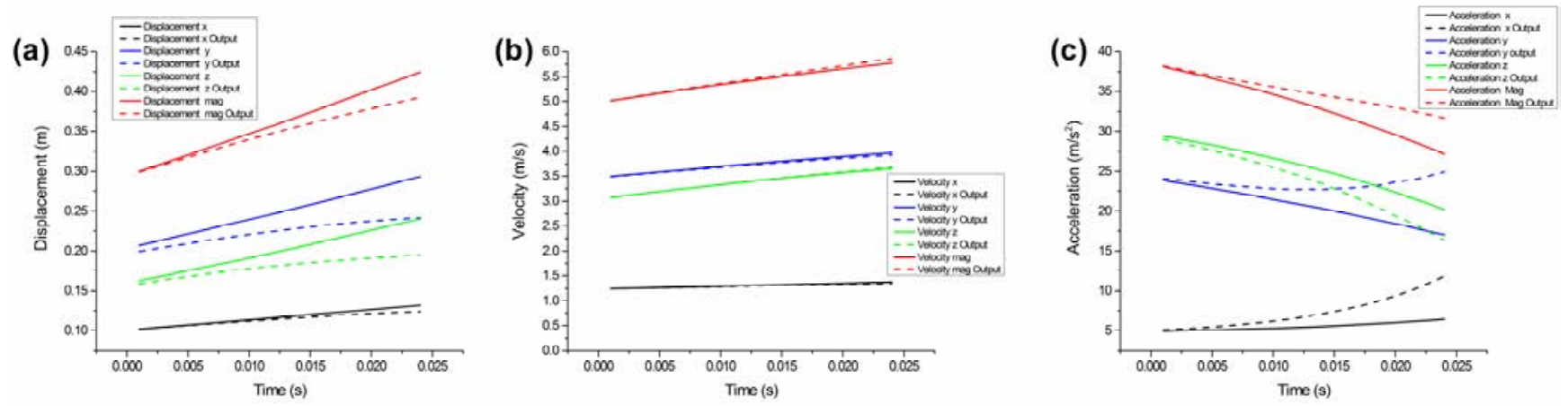

Figure 3. Original data and actual network output data of: (a) displacement ( $x, y, z$, and mag); (b) velocity (x, y, z, and mag); (c) acceleration ( $x, y$, z, and mag).

\section{Discussion}

Two criteria were used to assess the validity of the model for predicting kinematic parameters: first, the correlation between the network output data and the original analysis data was very high, $R>0.99$. This indicates that the results from the MLPs (predicted) were similar to those computed via integration of the ground reaction force in all tested variables (displacement $\mathrm{x}, \mathrm{y}, \mathrm{z}$; velocity $\mathrm{x}, \mathrm{y}, \mathrm{z}$; acceleration $\mathrm{x}, \mathrm{y}, \mathrm{z}$ ) (Table 1; Figure 3).

Second, the RMSE values for this neural model were the lowest for all predicted kinematic parameters during the network training $(70 \%$ training, $15 \%$ cross validation and $15 \%$ testing). In addition, the MAE was the lowest value at 0.002: 1.17 during training and testing of the MLPs. This means that the difference between network output data and the original analysis data was too small and the MLPs neural network was successfully predicting the target parameters with a high accuracy. It seems now we can use only the data from the force platform to predict the kinematic data in useful ways and it would be possible to deal with huge amount of the data coming from the force platform.

There are some advantages for using the MLPs neural network approach presented above: (1) it demonstrates the possibility of using the MLPs model to predict kinematic parameters at the take-off phase during the jump shot in handball, (2) It determines the correlation between network output data and the original analysis data, and (3) previous studies were dependent on the average of the parameters for all subjects whereas the presented method predicts kinematic parameters specific to a subject that is more accurate than the average with regards to kinematic parameters from all the subjects, especially when examining specific cases.

\section{Conclusions}

In conclusion, this study evaluated an MLP neural network approach for predicting kinematic parameters based on the output force plate parameters. Force, impulse, and work in three axes (x, y, z,) and magnitude (mag) were input parameters for the MLP neural network; and the target kinematic parameters were displacement, velocity, acceleration in three axes (x, y, z,) and magnitude (mag). The MLPs contained 12 processing elements, representing kinetic parameters, one hidden layer, and a "greedy search" output optimization. In all, $70 \%$ of all datasets were used to feed the MLPs neural network. The rest of the data, $30 \%$, were equally used for cross validation and to test the network model. The value of the mean square error (MSE) was very small suggesting the goodness of the prediction algorithm. In addition, there was a very strong correlation between network output data and the original data obtained via integration. Therefore, the use of the MLPs neural network is an accurate method for predicting kinematic parameters based on kinetic parameters in the handball jump shot, under the conditions of this study. And more studies are needed that investigate the validity and accurate of using the MLPs on other performance 
skills and biomechanical parameters.

\section{Acknowledgements}

We are grateful to Prof. Dr. Phillip Bishop, Department of Kinesiology, University of Alabama, United States of America. He moderated this paper and in that line improved the manuscript significantly.

\section{References}

[1] Wagner, H., et al., Individual and team performance in team-handball: a review. J Sports Sci Med, 2014. 13 (4): p. 808-16.

[2] Wagner, H., S. Kainrath, and E. Müller, Coordinative and tactical parameters of team-handball jump throw. The correlation of level of performance, throwing quality and selected techniquetactical parameters. Leistungssport, 2008. 38: p. 35-41.

[3] Gorostiaga, E. M., et al., Differences in physical fitness and throwing velocity among elite and amateur male handball players. International Journal of Sports Medicine 2005. 26 (3): p. 225-232.

[4] Wagner, H., et al., Kinematic description of elite vs. Low level players in team-handball jump throw. J Sports Sci Med, 2010.9 (1): p. 15-23.

[5] McKinon, W., et al., The agreement between reaction-board measurements and kinematic estimation of adult male human whole body centre of mass location during running. Physiological Measurement, 2004. 25 (6): p. 1339-1354.

[6] Mapelli, A., et al., Validation of a protocol for the estimation of three-dimensional body center of mass kinematics in sport. Gait Posture, 2014. 39 (1): p. 460-5.

[7] Payton, C. J. and R. M. Bartlett, Biomechanical Evaluation of Movement in Sport and Exercise. 2008, New York: Routledge.

[8] Zatsiorsky, V. M., Kinetics of Human Motion. 2002, Champaign: Human Kinetics.

[9] Multon, F., et al., Computer Animation of Human Walking: a Survey. J Visual Comp Animat, 1999. 10 (1): p. 39-54.

[10] Zeltzer, D., Motor control techniques for figure animation. IEEE Computer Graphics and Applications, 1982. 2 (9): p. 53-59.

[11] Arnaldi, B., et al. Animation control with dynamics. in In Proc. of Computer Animation. 1989.

[12] Witkin, A. and Z. Popovic. Motion warping. in Computer Graphics Proceedings, Annual Conference Series. 1995. Los Angeles: CMU.

[13] Ibarra, F. J. R., et al., Design of a Biomechanical Model and a Set of Neural Networks for Monitoring of Weightlifting. Research in Computing Science 2014. 80: p. 31-42.

[14] Saber-Sheikh, K., et al., Feasibility of using inertial sensors to assess human movement. Manual Ther, 2010. 15 (1): p. 122-125.

[15] Chau, T., A review of analytical techniques for gait data. Part 2: neural network and wavelet methods. Gait Posture, 2001. 13 (2): p. 102-20.
[16] Lapham, A. C. and R. M. Bartlett, The use of artificial intelligence in the analysis of sports performance: a review of applications in human gait analysis and future directions for sports biomechanics. J Sports Sci, 1995. 13 (3): p. 229-37.

[17] Verma, B. and C. Lane, Vertical jump height prediction using EMG characteristics and neural networks. Journal of Cognitive Systems Research, 2000. 1: p. 135-141.

[18] Favre, J., et al., A neural network model to predict knee adduction moment during walking based on ground reaction force and anthropometric measurements. J Biomech, 2012. 45 (4): p. 692-8.

[19] Rouhani, H., et al., Ambulatory assessment of $3 D$ ground reaction force using plantar pressure distribution. Gait Posture, 2010. 32 (3): p. 311-316.

[20] Bishop, C. M., Pattern Recognition and Machine Learning, ed. n. edition. 2007, New York: Springer. 738.

[21] Goulermas, J. Y., et al., Regression techniques for the prediction of lower limb kinematics. J Biomech Eng, 2005. 127 (6): p. 1020-4.

[22] Duda, R. O., P. E. Hart, and D. G. Stork, Pattern Classification, ed. n. edition. 2001, Hoboken: John Wiley and Sons. 654.

[23] Hassan, A., The Use of Modular Feed Forward Neural Networks in Anticipating the Results of Handball Championship 2015. American Journal of Sports Science, 2015. 3 (5): p. 93-97.

[24] Galajdová, A., D. Šimšík, and L. Madarász. Possibilities of gait parameters prediction from EMG data by Neural Networks in Computational intelligence: Proceedings of the 3nd international symposium of Hungarian researchers. 2002. Budapest: Polytechnic.

[25] Hahn, M. E., Feasibility of estimating isokinetic knee torque using a neural network model. J Biomech, 2007. 40 (5): p. $1107-1111$.

[26] Holzreiter, S., Autolabeling 3D tracks using neural networks. Clin Biomech, 2005. 20 (1): p. 1-8.

[27] Schollhorn, W. I., Applications of artificial neural nets in clinical biomechanics. Clin Biomech, 2004. 19 (9): p. 876-98.

[28] Begg, R. and J. Kamruzzaman, A machine learning approach for automated recognition of movement patterns using basic, kinetic and kinematic gait data. J Biomech, 2004. 38 (3): p. 401-408

[29] Wang, L. and T. S. Buchanan, Prediction of joint moments using a neural network model of muscle activations from EMG signals. IEEE Translations on Neural Systems and Rehabilitation Engineering 2002. 10 (1): p. 30-37.

[30] Liu, M. M., W. Herzog, and H. H. C. M. Savelberg, Dynamic muscle force prediction from EMG: an artificial neural network approach. J Electromyogr Kinesiol, 1999. 9 (6): p. 391-400.

[31] Oliver, G. D., H. A. Plummer, and D. W. Keeley, Muscle activation patterns of the upper and lower extremity during the windmill softball pitch. J Strength Cond Res, 2011.25 (6): p. 1653-8.

[32] Rojas, I. L., et al., Biceps activity during windmill softball pitching: injury implications and comparison with overhand throwing. Am J Sports Med, 2009. 37 (3): p. 558-65. 